\title{
An Assessment of Urinary Biomarkers in a Series of Declined Human Kidneys Measured During ex-vivo Normothermic Kidney Perfusion
}

\author{
Sarah A Hosgood, $\mathrm{PhD}^{1,2}$ Michael L Nicholson, DSc ${ }^{1,2}$ \\ ${ }^{1}$ Department of Surgery, Addenbrooke's Hospital, University of Cambridge. Cambridge CB2 \\ OQQ. UK \\ ${ }^{2}$ Department Infection, Immunity and Inflammation, Transplant Group, University of \\ Leicester. Leicester General Hospital. LE5 4PW. UK
}

Corresponding author

Dr Sarah A Hosgood

Senior Research Associate

Dept of Surgery,

University of Cambridge

Addenbrooke's Hospital

Level 9 PO BOX 202

Hill's Road

Cambridge

CB2 OQQ

UK

Tel +44 (0) 1223762002

Email:sh744@cam.ac.uk 


\section{Authorship page}

SAH: Designed the study, carried out the perfusion, wrote and reviewed the manuscript

MLN: Designed the study and reviewed the manuscript.

\section{Conflicts of Interest}

The authors declare no conflict of interest.

\section{Disclosures}

This study was supported by Kidney Research UK. The research was also funded by the National Institute for Health Research Blood and Transplant Research Unit (NIHR BTRU) in Organ Donation and Transplantation at the University of Cambridge in collaboration with Newcastle University and in partnership with NHS Blood and Transplant (NHSBT). The views expressed are those of the author(s) and not necessarily those of the NHS, the NIHR, the Department of Health or NHSBT.

We would like to thank Miss Meeta Patel for her technical help and Dr John Dormer for performing the histology analysis.

This is an open-access article distributed under the terms of the Creative Commons Attribution-Non Commercial-No Derivatives License 4.0 (CCBY-NC-ND), where it is permissible to download and share the work provided it is properly cited. The work cannot be changed in any way or used commercially without permission from the journal. 


\begin{abstract}
Background

The measurement of urinary biomarkers during ex-vivo normothermic kidney perfusion (EVKP) may aid in the assessment of a kidney prior to transplantation. This study measured levels of neutrophil gelatinase-associated lipocalin (NGAL), kidney injury molecule-1 (KIM1) and endothelin-1 (ET-1) during EVKP in a series of discarded human kidneys.
\end{abstract}

\title{
Methods
}

Fifty six kidneys from deceased donors were recruited into the study. Each kidney underwent 60 minutes of EVKP and was scored based on the macroscopic appearance, renal blood flow and urine output. The scores ranged from 1 (least injury) to 5 (most severe). Levels of oxygen consumption, extraction, creatinine fall and fractional excretion of sodium were measured during perfusion. Urinary levels of NGAL, KIM-1 and ET-1 were measured after EVKP.

\section{Results}

Thirty eight kidneys had an EVKP score of 1 or 2, 8 a score of 3 and 10 a score of 4 or 5 .

During EVKP lower levels of oxygen consumption, higher oxygen extraction, a lower decrement of serum creatinine and higher levels of NGAL and ET-1 were associated with a higher EVKP score $(\mathrm{P}<0.05)$. These parameters were also associated with a raised creatinine level in the donor before organ retrieval. Levels of KIM-1 were not associated with the perfusion parameters $(\mathrm{P}=0.649)$ or renal function in the donor $\left(\mathrm{R}^{2}=0.02458\right.$ : $\left.\mathrm{P}=0.271\right)$.

\section{Conclusion}

The measurement of urinary biomarkers, particularly NGAL in combination with functional perfusion parameters and the EVKP score provides an informative measure of kidney quality which may aid the decision to transplant the kidney. 


\section{Introduction}

Perfusion technologies provide a means to assess the quality of an organ prior to transplantation. This can aid in the decision process, preventing the likelihood of primary non function (PNF) and also the unnecessary discard of the organ. Hypothermic perfusion techniques have proved useful in determining the level of injury but perfusion parameters (flow and resistance) are poor predictors of outcome and cannot reliably discriminate between organs that should or should not be transplanted ${ }^{1}$.

Normothermic perfusion technologies have significant advantages over hypothermic techniques. Graft function is restored and the exposure to cold ischaemia is lessened. Furthermore, an assessment of functional parameters during perfusion not only allows a measure of injury but also provides an evaluation of recovery. We have previously described the application of an ex-vivo normothermic kidney perfusion (EVKP) quality assessment score in clinical transplantation ${ }^{2}$. The assessment score is based on 3 basic parameters during perfusion: Macroscopic appearance, renal blood flow and urine output. Kidneys are scored from 1 (least injury) to 5 (the most severe injury). Other markers of function and injury may also be incorporated into the assessment to provide a more comprehensive measure of quality.

Urinary biomarkers are emerging as an important diagnostic aid in the early detection of acute kidney injury (AKI) in a wide range of settings ${ }^{3-5}$. Neutrophil gelatinase-associated lipocalin (NGAL) and kidney injury molecule-1 (KIM-1) are 2 of the most recognised and reliable markers of kidney injury and point-of-care devices are available to provide results for both within 10 to 15 minutes. Endothelin-1 is a non-renal specific marker of tissue injury. Nevertheless, we have previously found it to be a reliable index of tubular injury in a porcine model $^{6,7}$. 
The aim of this study was to examine the relationship between urinary biomarkers [endothelin-1 (ET-1), neutrophil gelatinase-associated lipocalin (NGAL) and kidney injury molecule-1 (KIM-1)] and EVKP parameters in a series of human kidneys that were rejected for transplantation in the UK.

\section{Materials and Methods}

\section{Ethical approval}

From December 2012 to January 2014, 56 kidneys from the national organ allocation scheme that were deemed unsuitable for transplantation were recruited in this research project. Consent for the use of the organs for transplantation and research was obtained from the donor family by the Specialist Nurses in Organ Donation before organ retrieval. Ethical approval was granted for the study by the national research ethics commission in the UK, National Research Ethics System (12/EM/0143).

\section{Kidney Donor}

Kidneys were retrieved by the UK National Organ Retrieval Service as previously described ${ }^{2}$. The kidney donor information was recorded and included donor age, gender, donor type and terminal serum creatinine levels. The warm and cold ischaemic times were also recorded.

\section{Ex-vivo normothermic perfusion and functional analyses}

Kidneys were prepared for EVKP and the renal artery, vein and ureter cannulated.

The EVKP circuit was primed with a perfusate solution (Ringer's solution, Baxter

Healthcare) and supplements added to provide a physiological environment ${ }^{8,9}$. One unit of 
group 0 positive packed red blood cells from the local blood bank was added to the priming solution.

Kidneys were perfused with the red cell based solution at a set mean arterial pressure and near normal body temperature. Supplements were infused into the venous reservoir and arterial arm of the circuit to maintain normal homeostatic conditions as previously described $2,8-10$

Creatinine was added to the red cell based perfusate to allow an assessment of creatinine clearance and tubular function. The renal blood flow and mean arterial pressure were recorded continuously and the mean calculated. Urine output was collected throughout perfusion and the total output measured. Blood gas analysis of arterial and venous blood was used to record acid-base homeostasis and measure oxygen consumption and extraction. Blood samples were taken pre-perfusion and after 60 minutes of EVKP. A urine sample was collected after 60 minutes of EVKP. Percentage creatinine fall and fractional excretion of sodium were calculated and recorded.

\section{Urinary biomarkers}

Urine levels of endothelin 1 (ET-1) were determined using an enzyme immunometric assay kit (Assay Design. Michigan. USA). Levels of NGAL and KIM-1 were measured using a Human NGAL sandwich enzyme-linked immunosorbent assay (ELISA) kit (BioPorto Diagnostics, Gentofte. Denmark) and KIM-1 ELISA kit (Enzo Life Sciences, Exeter, UK). The samples and standards were added in duplicate to the precoated plates and the assays carried out as per the manufacturer's instructions. 


\section{EVKP assessment parameters}

\section{Macroscopic assessment}

Each kidney was categorised into 3 groups according to its macroscopic appearance during EVKP as follows ${ }^{2}$.

Grade I: Excellent perfusion (global pink appearance - 1 point)

Grade II: Moderate perfusion (patchy pink/purple appearance which either remained or improved during EVKP - 2 points)

Grade III: Poor perfusion (global mottling and purple/black appearance which remained throughout EVKP - 3 points).

\section{Derivation of EVKP assessment score}

A combination of the macroscopic and functional parameters were used to create an index of organ quality. Receiver operating characteristic (ROC) curves were previously used to determine thresholds of renal blood flow and urine output to differentiate between macroscopic grades I and II versus grade III ${ }^{8}$. These thresholds were combined with the macroscopic grade to give an overall EVKP assessment score of 1 to 5. Macroscopic grades I, II and III were assigned points of 1, 2 and 3, respectively. Kidneys were assessed by 2 assessors blinded to the donor details. Kidneys with a mean renal blood flow below the threshold $(<50 \mathrm{ml} / \mathrm{min} / 100 \mathrm{~g})$ were given an additional score of 1 . Kidneys producing less than the threshold of urine output ( $<43 \mathrm{ml}$ in 60 minutes) were also given an additional score of 1 . Therefore, overall EVKP assessment scores ranged from 1 indicating the least injury to 5, the most severe. This score provides a quantitative measure of functional parameters that supports the macroscopic appearance. 


\section{Histological evaluation}

A wedge renal biopsy was taken on arrival at the laboratory after the period of static cold storage. The tissue was fixed in $10 \%$ formal saline then embedded in paraffin wax. Sections from the paraffin embedded tissue were cut $(4 \mu \mathrm{m})$ and stained with Haematoxylin \& Eosin for histopathological scoring.

A consultant pathologist graded the sections using the Remuzzi score and assessed the level of acute tubular injury (ATI). Sections were graded mild, mild to moderate, moderate and severe for the presence of ATI.

\section{Statistics}

Continuous data are presented as mean \pm SD. Data was compared using Kruskal Wallis test with Dunn's multiple comparisons test were appropriate. Categorical variables were analysed by Chi-squared. $\mathrm{P}<0.050$ was considered statistically significant. Donor factors (age, retrieval creatinine, the warm ischaemic time and cold ischaemic time) and perfusion parameters (The EVKP score, renal blood flow, oxygen consumption, oxygen extraction, urine output, percentage creatinine fall and fractional excretion of sodium) were correlated with the urinary biomarkers score using linear regression (Pearsons). The kidney donor risk index (KDRI) was calculated ${ }^{11}$ and correlated with the EVKP score. GraphPad Prism 6 was used for statistical analysis (GraphPad Software, La Jolla, California, USA).

\section{Results}

Fifty six kidneys from 41 donors were included in the study. The kidneys were declined for transplantation due to the following reasons. Donor history $(\mathrm{n}=12)$, poor in-situ flush $(\mathrm{n}=9)$, hypothermic machine perfusion parameters $(\mathrm{n}=6)$, anatomical/technical reasons $(n=14)$, suspected malignancy $(n=6)$, acute kidney injury $(A K I)(n=1)$, histology 
score $(n=3)$, fat embolism $(n=2)$, infection $(n=1)$, prolonged cold ischaemia $(n=1)$ and no suitable recipient $(\mathrm{n}=1)$. The EVKP scores were as follows: Twenty two scored 1, 16 scored 2, 8 scored 3, 4 scored 4 and 6 scored 5. To simplify analysis, the kidneys were grouped as follows: Group A, EVKP score 1 or $2(\mathrm{n}=38)$, Group B, EVKP score $3(\mathrm{n}=8)$ and Group C, EVKP score 4 or $5(n=10)$.

\section{Donor demographics}

There were significantly fewer female donors in the EVKP group B and C kidneys compared to group A $(\mathrm{P}=0.034$; Table 1$)$. In the EVKP group $\mathrm{B}$ kidneys all the donors where from DBD donors, whereas in EVKP group $\mathrm{C}$, all where from $\mathrm{DCD}$ donors $(\mathrm{P}=0.001$; Table 1). There was no significant difference in the duration of warm or cold ischaemia $(\mathrm{P}=$ 0.581, 0.051, respectively). The terminal serum creatinine was significantly higher in EVKP group $\mathrm{C}$ kidneys compared to group $\mathrm{A}(\mathrm{P}=0.001$; Table 1$)$. Nine out of the 10 donors in EVKP group C kidneys had a raised terminal serum level $(>121 \mu \mathrm{mol} / \mathrm{L})$.

\section{Perfusion Parameters and Urinary Biomarkers}

Kidneys in EVKP group A had improved perfusion parameters compared to groups B and C. The renal blood flow, oxygen consumption and urine output were significantly higher in group A compared to groups $\mathrm{B}$ and $\mathrm{C}(\mathrm{P}<0.05$; Table 2$)$. Serum creatinine fall was also significantly greater in group A compared to the group $\mathrm{C}(\mathrm{P}<0.05$; Table 2$)$. There was no significant difference in the level of fractional excretion of sodium between the groups although, levels were numerically higher in group $\mathrm{B}$ and $\mathrm{C}$ kidneys $(\mathrm{P}=0.317$; Table 2$)$.

Levels of ET-1 were significantly higher in EVKP group B kidneys compared to the A (P = 0.008; Figure 1a). Although, numerically levels were the highest in EVKP group C kidneys. NGAL was significantly lower in EVKP group A kidneys compared to group $\mathrm{C}(\mathrm{P}=0.018$; 
Figure 1b). There was no significant difference in the urinary levels of KIM-1 between the groups $(\mathrm{P}=0.649 ;$ Figure $1 \mathrm{c})$.

Higher urinary levels of ET-1 and NGAL were associated a higher EVKP assessment score $\left(\mathrm{R}^{2}=0.1771 ; \mathrm{P}=0.001, \mathrm{R}^{2}=0.0997 ; \mathrm{P}=0.018\right.$, respectively). There was no correlation with the assessment score and KIM-1 $\left(\mathrm{R}^{2}=0.00532 ; \mathrm{P}=0.600\right)$.

Lower urinary levels of NGAL were associated with a higher mean renal blood flow $(\mathrm{P}=$ $\left.0.008 ; R^{2} 0.1890\right)$, higher level of oxygen consumption $\left(P=0.004 ; R^{2}=0.1440\right)$, increased urine output $\left(\mathrm{P}=0.049 ; \mathrm{R}^{2}=0.070\right)$ and decrement in creatinine $\left(\mathrm{P}=0.0013 ; \mathrm{R}^{2}=0.1850\right)$. Lower urinary levels of ET-1 were associated with a greater decrement in creatinine $(\mathrm{P}=$ $\left.0.001 ; \mathrm{R}^{2}=0.1938\right)$. There was no significant correlation between the urinary levels of KIM1 and any of the perfusion parameters.

\section{Correlation of perfusion parameters with donor demographics}

There was no association with the donor age or length of warm or cold ischaemia and any of the perfusion parameters $(\mathrm{P}>0.05)$. Kidneys from female donors were associated with higher urine output $\left(\mathrm{P}=0.0001 ; \mathrm{R}^{2}=0.2367\right)$, a greater decrement in creatinine $(\mathrm{P}<0.0001$; $\left.\mathrm{R}^{2}=0.2624\right)$ and a lower EVKP score $\left(\mathrm{P}=0.001 ; \mathrm{R}^{2}=0.1528\right)$. A higher serum creatinine level before retrieval was associated a lower level of renal blood flow, oxygen consumption, higher oxygen extraction and a lower level of renal function during EVKP ( $\mathrm{P}>0.05$; Table 3$)$. It was also associated with a higher EVKP score. There was no association with the level of fractional excretion of sodium and any of the donor demographics $(\mathrm{P}=0.4822$; Table 3$)$. The KDRI score did not correlate with the EVKP score $\left(\mathrm{R}^{2}=0.0010 ; \mathrm{P}=0.820\right)$. 


\section{Correlation of ET-1, NGAL and KIM-1 with donor demographics}

Higher levels of urinary ET-1 and NGAL were associated with a raised creatinine before retrieval in the donor $\left(\mathrm{R}^{2}=0.1139 ; \mathrm{P}=0.014\right.$ and $\mathrm{R}^{2}=0.1385 ; \mathrm{P}=0.007$, respectively). However, there was no association with donor age, warm ischaemic time or the cold ischaemic time $(\mathrm{P}>0.05)$. There was no association with KIM-1 and donor demographics or creatinine levels before retrieval $\left(\mathrm{R}^{2}=0.02458 ; \mathrm{P}=0.272\right)$.

\section{Donation after Circulatory Death versus Donation after Brain Death Kidneys}

The cold ischaemic time was significantly longer in the DBD kidneys compared to the DCD (P 0.026; Table 4). Urinary levels of ET-1 were significantly lower in the DCD kidneys compared to DBD ( $\mathrm{P}=0.043$; Table 4). There were no other significant differences between the donor demographics, perfusion parameters or urinary biomarkers (Table 4).

\section{Histology}

Thirty two biopsies were available from EVKP group A kidneys, all of group B kidneys and from 7/10 of group C kidneys. The median Remuzzi score was 4 (range 0-10) in EVKP group A kidneys, 5 in group B (range 1-10) and 3 (range 0-6) in group C kidneys. Eleven out of 32 of EVKP group A kidneys had mild ATI, 5 mild to moderate and 2 moderate ATI. One kidney in EVKP group B had mild, 1 mild to moderate and 1 moderate ATI. Two kidneys in EVKP group C kidneys had mild ATI and 2 had moderate ATI. There was no association with urinary levels of NGAL, KIM-1, ET-1 or any of the perfusion parameters, the Remmuzi score or the level of AKI $(\mathrm{P}>0.05)$. 


\section{Discussion}

This study demonstrates that levels of urinary ET-1 and NGAL measured after 60 minutes of EVKP were significantly correlated with perfusion parameters during EVKP. These biomarkers and EVKP perfusion parameters were also significantly correlated with terminal renal function in the donor. There was no association between urinary KIM-1 after EVKP and either perfusion parameters or terminal renal function in the donor. The measurement of biomarkers during EVKP provides additional information about donor organ quality.

Normothermic perfusion techniques are evolving as an important asset in the decision process to determine the suitability of an organ for transplantation. We have previously reported that basic functional parameters (renal blood flow and urine output) together with an assessment of the macroscopic appearance can be used to formulate a kidney quality score ${ }^{2}$. Evidence so far suggests that a higher EVKP score is associated with an increased risk of DGF ${ }^{2}$. This technology has also been used to assess human kidneys declined for transplantation due to poor in-situ perfusion at the time of retrieval ${ }^{10}$. We have subsequently gone onto to successfully transplant 2 of these kidneys based on this assessment ${ }^{12}$. This present study has shown that other measures of function including the rate of oxygen consumption, oxygen extraction and creatinine fall can be used to provide a more detailed assessment of function and quality.

Measurements of serum creatinine are used clinically to assess the level of renal function. Nonetheless, creatinine levels are affected by other physiological functions and are regarded as a late marker of renal injury ${ }^{4}$. Urinary biomarkers, particularly NGAL and KIM1 are sensitive measures of kidney injury and can be used to provide a more accurate assessment ${ }^{4-6,13,14}$. In the posttransplant setting, they have a high predictive value and are 
strongly associated with graft dysfunction. More recently Koo et al measured several biomarkers [NGAL, KIM-1 and liver fatty acid binding protein (L-FABP)] in a series of 94 deceased donors to determine kidney quality and outcome ${ }^{15}$. They used a multiple logistic regression model to generate a prediction score. Independently, NGAL and L-FABP were associated with AKI in the donor and predictive of reduced graft function in the recipient [area under the curve receiver operating characteristics (AUC ROC) 0.758 and 0.704, respectively]. When included in a scoring system their predictive value was greater (AUC ROC 0.808). In a larger series of 1304 deceased donors, Reese et al also found that higher levels of urinary NGAL were strongly associated with donor AKI and had some association with DGF in the recipient ${ }^{16}$. However, their ability to predict PNF and determine which organs should be transplanted or discarded was low. Hollmen et al showed that in donors with a urinary NGAL $\geq 18 \mathrm{ng} / \mathrm{ml}$, DGF was more prolonged and long term outcome reduced. Interestingly, in all these studies KIM-1 had no association with donor AKI or outcome ${ }^{17}$. NGAL is an earlier marker of kidney injury upregulated within hours whereas KIM-1 is reported as a later marker, although the literature is conflicting ${ }^{18}$. van den Akker et al found that KIM-1 was not detectable in the perfusate of the graft after preservation or until day 4 post-transplant ${ }^{19}$. In contrast, Field et al recently reported that KIM-1 was a promising marker of AKI in a series of deceased donors ${ }^{20}$. Biomarkers have also been used during hypothermic machine perfusion in combination with flow and resistance parameters to determine quality. However, individually they had low a predictive value and could not distinguish between transplanted and discarded kidneys ${ }^{21}$.

The third biomarker used in this study ET-1, is involved in regulating renal vascular tone and tubular secretion of electrolytes and water. Clinically, high urinary levels have been associated with chronic kidney ${ }^{22,23}$ and polycystic kidney disease ${ }^{24}$. In a porcine model of reperfusion injury, we previously found that elevated levels of ET-1 accurately reflected the 
level of ischaemic injury ${ }^{6,7}$. More recently, ET-1 has been used as a marker of lung injury during ex-vivo lung perfusion (EVLP) ${ }^{25}$. Perfusate levels predicted lung function and were associated with outcome after transplantation.

Levels of fractional excretion of sodium a marker of tubular function and urinary KIM-1 were not significantly different across the grades of kidneys and not associated with donor AKI. Therefore, their use in this setting appears to be limited. The histology revealed a wide variation in the level of donor related damaged. A significant proportion of kidneys had evidence of acute kidney injury. Renal function is not necessarily associated with histological findings ${ }^{25}$ and we found no significant correlation between the Remuzzi score, urinary biomarkers, donor demographics or EVKP parameters. This is in agreement with Koo et al who found no association with the biopsy injury score, NGAL or outcome in their series of deceased donor kidneys ${ }^{15}$. The Remuzzi score is a histological assessment of chronic renal damage, whereas EVKP provides functional information about acute tubular injury. The lack of correlation between the 2 is therefore unsurprising. This argument applies equally to the KDRI.

The role of urinary biomarkers has not been previously investigated during EVKP of human kidneys. Although there is no follow up data on outcome, this study provides important information for the future development of EVKP as an assessment device. Our described technique of EVKP is carried out at the end of the preservation interval for 60 minutes prior to transplantation. The technique requires some added resources but is straightforward and brief. We found that kidneys with a higher EVKP score, indicating lower quality also had reduced functional parameters and higher levels of urinary NGAL and ET-1. These parameters were also associated with raised levels of serum creatinine recorded in the donor before organ retrieval. Whilst this suggests that these biomarkers are reliable indices of acute tubular injury which may aid in the assessment of a kidney, it also raises the question of 
the cost effectiveness and extra utility of measuring biomarkers in addition to the currently available donor data and EVKP assessment. The biomarkers are typically measured using ELISA techniques. Although, a point-of-care devise is available for NGAL which would makes its application into clinical practice relatively straightforward.

Larger series are required to determine whether these biomarkers can be used as a reliable assessment tool in combination with EVKP to predict graft outcome. We are currently performing a multicentre randomised controlled trial comparing static cold storage with EVKP in DCD kidney transplantation which is due to be completed in 2020 (ISRCTN15821205). Urine samples are being collected during EVKP $(n=200)$ for the analysis of a range of biomarkers including NGAL, ET-1 and L-FABP. These will be correlated with transplant graft outcome, donor demographics, EVKP parameters and histological analysis. This is likely to provide us with a more conclusive evaluation of their use in this setting.

A limitation to the described EVKP technique is perhaps the short duration. Of note during the 60 minutes of perfusion, there was no association between any of the perfusion parameters or urinary biomarkers and the level of ischaemic injury. This may be explained by the duration and conditions necessary for these effects to become evident. Warm and cold ischaemic injury have a detrimental effect on graft function and outcome. The re-introduction of oxygenated blood mediates a series of injury processes involving activated endothelial cells causing vasoconstriction, the recruitment of leukocytes to the injured tissue leading to failure of the microcirculation and the 'no-reflow' phenomena ${ }^{27}$. There is also a release of damage associated molecular pattern molecules which activate reactive oxygen species, molecular and signalling cascades ${ }^{28}$. These events cause significant tissue injury which results in AKI and DGF. Although circulation is restored during EVKP, the conditions are 
designed to be protective. The absence of white cells, platelets and complement factors abrogate graft injury and therefore it perhaps unsurprising that the impact of ischaemic injury appears to be undetected during this time. Nonetheless, longer perfusion times may provide additional information on the impact of ischaemic injury.

In conclusion, EVKP continues to be developed as a comprehensive pretransplant assessment technology/tool. The measurement of urinary biomarkers during EVKP undoubtedly provides additional information but it is not clear at this point whether or not this will improve our ability to select marginal kidneys for transplantation. As this was a preliminary study of a relatively small number of discarded human kidneys, further analysis from the randomised controlled trial will evaluate the potential value of biomarkers in predicting clinical allograft outcomes. 


\section{Acknowledgments}

We would like to thank Miss Meeta Patel for her technical help and Dr John Dormer for performing the histology analysis. 


\section{References}

1: Jochmans I, Moers C, Smits JM, Leuvenink HG, Treckmann J, Paul A, Rahmel A, Squifflet JP, van Heurn E, Monbaliu D, Ploeg RJ, Pirenne J. The prognostic value of renal resistance during hypothermic machine perfusion of deceased donor kidneys. Am $J$ Transplant. 2011;11(10):2214-20.

2: Hosgood SA, Barlow AD, Hunter JP, Nicholson ML. Ex vivo normothermic perfusion for quality assessment of marginal donor kidney transplants. Br J Surg. 2015;102(11):143340.

3: Aydoğdu M, Gürsel G, Sancak B, Yeni S, Sarı G, Taşyürek S, Türk M, Yüksel S, Senes M, Ozis TN. The use of plasma and urine neutrophil gelatinase associated lipocalin (NGAL) and Cystatin C in early diagnosis of septic acute kidney injury in critically ill patients. Dis Markers. 2013;34(4):237-46.

4: Parikh CR, Devarajan P. New biomarkers of acute kidney injury. Crit Care Med. 2008 ; 36: p. S159-65.

5: Vincent IS, Okusa MD. Biology of renal recovery: molecules, mechanisms, and pathways. Nephron Clin Pract. 2014;127(1-4):10-4.

6: Patel M, Hosgood S, Nicholson ML. The effects of arterial pressure during normothermic kidney perfusion. J Surg Res. 2014;191(2):463-8.

7: Hosgood SA, Bagul A, Nicholson ML. Minimising cold ischaemic injury in an experimental model of kidney transplantation. Eur J Clin Invest. 2011 ;41(3):233-40.

8: Nicholson ML, Hosgood SA. Renal transplantation after ex vivo normothermic perfusion: the first clinical study. Am J Transplant. 2013;13(5):1246-52.

9: Hosgood SA, Nicholson ML. First in man renal transplantation after ex vivo normothermic perfusion. Transplantation. 2011;92(7):735-8. 
10: Hosgood SA, Barlow AD, Dormer J, Nicholson ML. The use of ex-vivo normothermic perfusion for the resuscitation and assessment of human kidneys discarded because of inadequate in situ perfusion. J Transl Med. 2015;13:329.

11: https://optn.transplant.hrsa.gov/resources/allocation-calculators/kdpi-calculator/ (accessed $02 / 08 / 2016)$

12: Hosgood SA, Saeb-Parsy K, Hamed MO, Nicholson ML. Successful transplantation of human kidneys deemed untransplantable but resuscitated by ex-vivo normothermic machine perfusion. [published online ahead of print June 7, 2016]. Am J Transplant. doi: 10.1111/ajt.13906.

13: Mishra J, Dent C, Tarabishi R, et al. Neutrophil gelatinase-associated lipocalin (NGAL) as a biomarker for acute renal injury after cardiac surgery. Lancet. 2005; 365: p. 12311238.

14: Hollmen ME, Kyllönen LE, Inkinen KA, Lalla ML, Merenmies J, Salmela KT. Deceased donor neutrophil gelatinase-associated lipocalin and delayed graft function after kidney transplantation: a prospective study. Crit Care. 2011;15(3):R121.

15: Koo TY, Jeong JC, Lee Y, Ko KP, Lee KB, Lee S, Park SJ, Park JB, Han M, Lim HJ, Ahn C, Yang J. Pre-transplant Evaluation of Donor Urinary Biomarkers can Predict Reduced Graft Function After Deceased Donor Kidney Transplantation. Medicine (Baltimore). 2016;95(11):e3076.

16: Reese PP, Hall IE, Weng FL, Schröppel B, Doshi MD, Hasz RD, Thiessen-Philbrook H, Ficek J, Rao V, Murray P, Lin H, Parikh CR. Associations between Deceased-Donor Urine Injury Biomarkers and Kidney Transplant Outcomes. $J$ Am Soc Nephrol. 2016;27(5):1534-43. 
17: Hollmen ME, Kyllönen LE, Merenmies J, Salmela KT. Serum neutrophil gelatinaseassociated lipocalin and recovery of kidney graft function after transplantation. $B M C$ Nephrol. 2014;15:123.

18: Parikh CR, Thiessen-Philbrook H, Garg AX, Kadiyala D, Shlipak MG, Koyner JL, Edelstein CL, Devarajan P, Patel UD, Zappitelli M, Krawczeski CD, Passik CS, Coca SG; TRIBE-AKI Consortium. Performance of kidney injury molecule-1 and liver fatty acid-binding protein and combined biomarkers of AKI after cardiac surgery. Clin J Am Soc Nephrol. 2013;8(7):1079-88.

19: van den Akker EK, Hesselink DA, Manintveld OC, IJzermans JN, de Bruijn RW, Dor FJ. Neutrophil Gelatinase-Associated Lipocalin, but Not Kidney Injury Marker 1, Correlates with Duration of Delayed Graft Function. Eur Surg Res. 2015;55(4):319-327.

20: Field M, Dronavalli V, Mistry P, Drayson M, Ready A, Cobbold M, Inston N. Urinary biomarkers of acute kidney injury in deceased organ donors--kidney injury molecule-1 as an adjunct to predicting outcome. Clin Transplant. 2014;28(7):808-15.

21: Parikh CR, Hall IE, Bhangoo RS, Ficek J, Abt PL, Thiessen-Philbrook H, Lin H, Bimali M, Murray PT, Rao V, Schröppel B, Doshi MD, Weng FL, Reese PP. Associations of Perfusate Biomarkers and Pump Parameters with Delayed Graft Function and Deceased Donor Kidney Allograft Function. Am J Transplant. 2016;16(5):1526-39.

22: Grenda R, Wühl E, Litwin M, Janas R, Sladowska J, Arbeiter K, Berg U, Caldas-Afonso A, Fischbach M, Mehls O, Sallay P, Schaefer F; ESCAPE Trial group. Urinary excretion of endothelin-1 (ET-1), transforming growth factor- beta1 (TGF-beta1) and vascular endothelial growth factor (VEGF165) in paediatric chronic kidney diseases: results of the ESCAPE trial. Nephrol Dial Transplant. 2007;22(12):3487-94.

23: Barton M, Sorokin A. Endothelin and the glomerulus in chronic kidney disease. Semin Nephrol. 2015;35(2):156-67. doi: 10.1016/j.semnephrol.2015.02.005. Review. 
24: Raina R, Lou L, Berger B, Vogt B, Do AS, Cunningham R, Vasavada P, Herrmann K, Dell K, Simonson M. Relationship of urinary endothelin-1 with estimated glomerular filtration rate in autosomal dominant polycystic kidney disease: a pilot cross-sectional analysis. BMC Nephrol. 2016;17:22.

25: Machuca TN, Cypel M, Zhao Y, Grasemann H, Tavasoli F, Yeung JC, Bonato R, Chen M, Zamel R, Chun YM, Guan Z, de Perrot M, Waddell TK, Liu M, Keshavjee S. The role of the endothelin-1 pathway as a biomarker for donor lung assessment in clinical ex vivo lung perfusion. J Heart Lung Transplant. 2015;34(6):849-57.

26: Re L, Cicora F, Petroni J, Goldberg J, Rial MC, Casadei D. Comparison between clinical and histopathological scoring in cadaveric kidney transplantation and its correlation with posttransplant evolution. Transplant Proc. 2006 ;38(3):903-4.

27: Basile DP, Yoder MC. Renal endothelial dysfunction in acute kidney ischemia reperfusion injury. Cardiovasc Hematol Disord Drug Targets. 2014;14(1):3-14. Review.

28: Doi K, Ishizu T, Tsukamoto-Sumida M, Hiruma T, Yamashita T, Ogasawara E, Hamasaki Y, Yahagi N, Nangaku M, Noiri E. The high-mobility group protein B1-Tolllike receptor 4 pathway contributes to the acute lung injury induced by bilateral nephrectomy. Kidney Int. 2014; 86(2):316-26. 


\section{Figure Legends}

Figure 1a: Urinary levels of endothelin-1 (ET-1) in EVKP group A, B and C kidneys after 60 minutes of ex-vivo normothermic perfusion (EVKP). ( $\mathrm{P}=0.008$ Group A and B versus $\mathrm{C})$.

Figure 1b: Urinary levels of neutrophil gelatinase-associated lipocalin (NGAL) in EVKP group $\mathrm{A}, \mathrm{B}$ and $\mathrm{C}$ kidneys after 60 minutes of ex-vivo normothermic perfusion $(\mathrm{EVKP}) .(\mathrm{P}=$ 0.018 Group A versus B and C).

Figure 1c: Urinary levels of kidney injury molecule - 1 (KIM-1) in EVKP group A, B and C kidneys after 60 minutes of ex-vivo normothermic perfusion (EVKP). (P=0.649). 


\section{Figure 1}
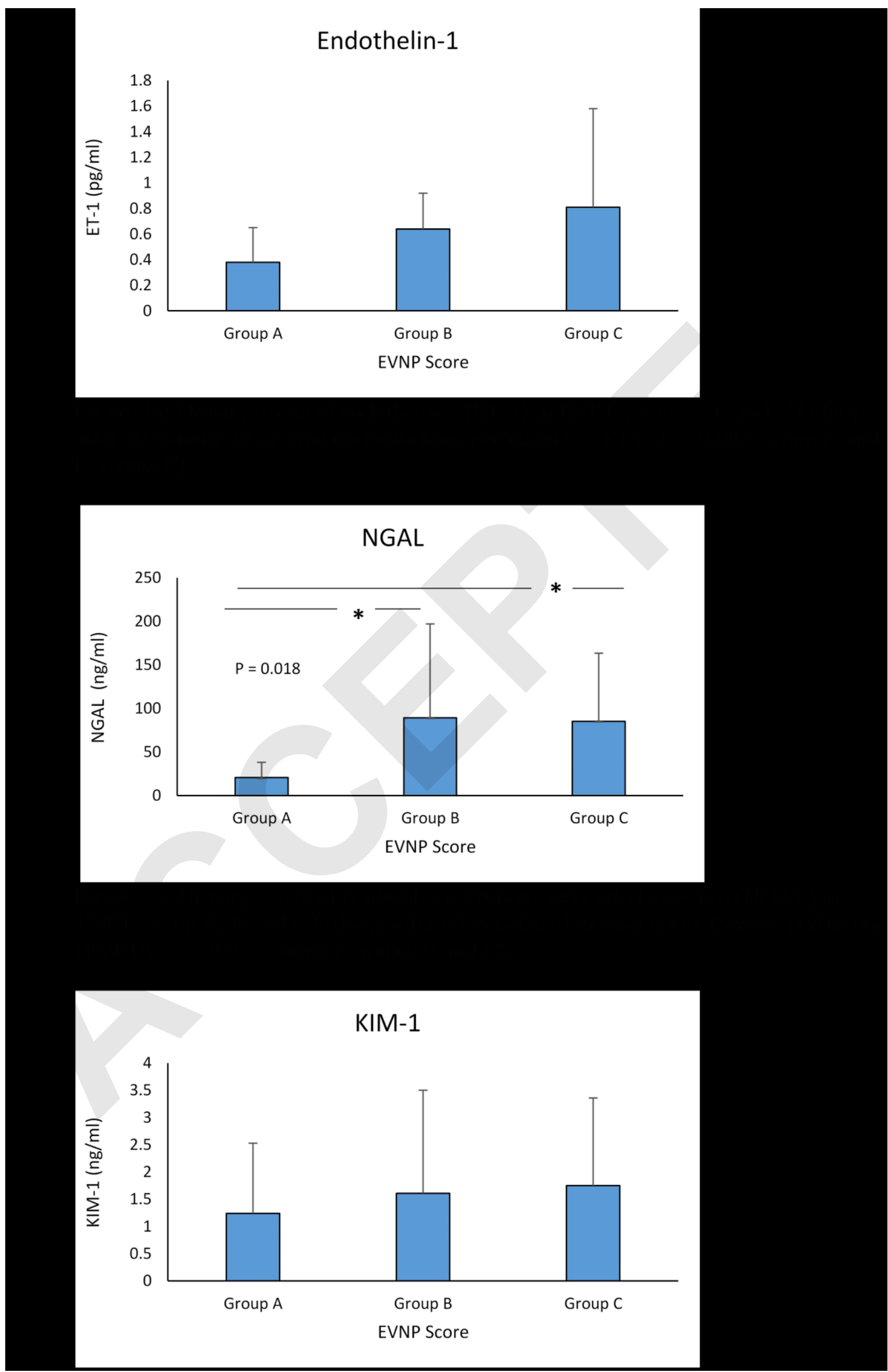
Table 1: The mean donor age, gender, donor type; donation after circulatory death (DCD), donation after brain death (DBD), warm ischaemic time (WIT), cold ischaemic time (CIT), retrieval creatinine $(\mathrm{Cr})$ and median Kidney donor risk index (KDRI) percentage. (* EVKP group A versus group B).

\begin{tabular}{|c|c|c|c|c|}
\hline EVKP Score & $\begin{array}{c}\text { Group A } \\
(\mathrm{n}=38)\end{array}$ & $\begin{array}{l}\text { Group B } \\
(\mathrm{n}=8)\end{array}$ & $\begin{array}{l}\text { Group C } \\
(\mathrm{n}=10)\end{array}$ & $P$ value \\
\hline $\operatorname{Age}(y)$ & $61 \pm 11$ & $66 \pm 14$ & $54 \pm 16$ & 0.162 \\
\hline $\mathbf{M}: \mathbf{F}$ & $17: 21$ & $5: 3$ & $9: 1$ & 0.034 \\
\hline DCD : DBD & $23: 15$ & $0: 8$ & $10: 0$ & 0.001 \\
\hline WIT (min) & $13.1 \pm 4$ & & $12.5 \pm 3.6$ & 0.581 \\
\hline CIT (h.min) & $32.2 \pm 13$ & $35 \pm 19$ & $22.7 \pm 7.0$ & 0.051 \\
\hline Retrieval Cr $(\mu \mathrm{mol} / \mathrm{L})$ & $88 \pm 44$ & $139 \pm 87$ & $233 \pm 126$ & $0.001 *$ \\
\hline KDRI (\%) & $86(20-100)$ & $96(53-100)$ & $71(62-100)$ & 0.311 \\
\hline
\end{tabular}


Table 2: The mean renal blood flow, oxygen consumption, oxygen extraction, total urine output, serum creatinine fall and fractional excretion of sodium (Fr ex $\mathrm{Na}+$ ) during ex-vivo normothermic perfusion (EVKP) in kidneys with an EVKP score of 1 to 5. (* EVKP group A versus $\mathrm{C} ; * *$ EVKP group A versus B and C).

\begin{tabular}{|c|c|c|c|c|}
\hline EVKP Score & $\underset{(\mathrm{n}=38)}{\text { Group A }}$ & $\begin{array}{l}\text { Group B } \\
(\mathrm{n}=8)\end{array}$ & $\begin{array}{l}\text { Group C } \\
(\mathrm{n}=10)\end{array}$ & $P$ value \\
\hline $\mathrm{RBF}(\mathrm{ml} / \mathrm{min} / \mathbf{1 0 0 g})$ & $86.1 \pm 27.4$ & $51.3 \pm 21.5$ & $37.9 \pm 19.1$ & $<0.0001 * *$ \\
\hline $\begin{array}{l}O_{2} \text { Consumption } \\
(\mathrm{ml} / \mathrm{min} / \mathrm{g})\end{array}$ & $60.7 \pm 21.7$ & $36.7 \pm 16.5$ & 30.0 & $0.001 * *$ \\
\hline $\mathrm{O}_{2}$ Extraction (\%) & $7.37 \pm 6.20$ & 10.04 & $13.5 \pm 11.1$ & 0.139 \\
\hline Urine output (ml) & $121 \pm 70$ & & $26 \pm 20$ & $0.001 *$ \\
\hline Cr Fall (\%) & $48.1 \pm 7.9$ & $36.0 \pm 17.2$ & $30.7 \pm 6.5$ & $0.001 *$ \\
\hline $\mathrm{Fr}$ ex $\mathrm{Na}+(\%)$ & $52.4 \pm 21.5$ & $63.9 \pm 22.0$ & $67.6 \pm 35.7$ & 0.317 \\
\hline
\end{tabular}


Table 3: Pearson correlation with the terminal serum creatinine in the donor and the mean renal blood flow, oxygen consumption, oxygen extraction, total urine output, serum creatinine fall, fractional excretion of sodium (Fr ex $\mathrm{Na}+$ ) and EVKP quality assessment score during ex-vivo normothermic perfusion (EVKP).

\begin{tabular}{|c|c|c|}
\hline & $\mathbf{R}^{2}$ & P value \\
\hline RBF (ml/min/100g) & 0.1514 & 0.004 \\
\hline $\mathrm{O}_{2}$ Consumption $(\mathrm{ml} / \mathrm{min} / \mathrm{g})$ & 0.1653 & 0.003 \\
\hline $\mathrm{O}_{2}$ Extraction (\%) & 0.1295 & 0.011 \\
\hline Urine output (ml) & 0.1798 & 0.002 \\
\hline Cr Fall (\%) & 0.1230 & $<0.0001$ \\
\hline $\mathrm{Fr}$ ex $\mathrm{Na}+(\%)$ & 0.1013 & 0.4822 \\
\hline EVKP Score & 0.4201 & $<0.0001$ \\
\hline
\end{tabular}


Table 4: Donor demographics, perfusion parameters and urinary biomarkers in donation after circulatory death and donation after brain death donors.

\begin{tabular}{|c|c|c|c|}
\hline & DCD & DBD & P Value \\
\hline Donor age (y) & $60.2 \pm 12.0$ & $61.5 \pm 14.3$ & 0.721 \\
\hline $\mathbf{M}: \mathbf{F}$ & $22: 11$ & $9: 14$ & 0.057 \\
\hline WIT (min) & $12.9 \pm 4.1$ & & \\
\hline CIT (h.min) & $27.1 \pm 10.7$ & $36.5 \pm 16.0$ & 0.026 \\
\hline Retrieval Creatinine $(\mu \mathrm{mol} / \mathrm{L})$ & $139 \pm 106$ & $104 \pm 61$ & 0.292 \\
\hline Renal Blood Flow (ml/min/100g) & $75.7 \pm 36.3$ & $68.1 \pm 25.3$ & 0.391 \\
\hline Oxygen Consumption $(\mathrm{ml} / \mathrm{min} / \mathrm{g})$ & $51.9 \pm 24.4$ & $51.7 \pm 24.6$ & 0.986 \\
\hline Oxygen Extraction (\%) & $8.7 \pm 8.5$ & $9.41 \pm 6.3$ & 0.733 \\
\hline Urine output (ml) & $93 \pm 74$ & $112 \pm 81$ & 0.360 \\
\hline Fractional Excretion of $\mathrm{Na}+(\%)$ & $59.1 \pm 24.4$ & $53.7 \pm 23.0$ & 0.438 \\
\hline Endothelin-1 (pg/ml) & $0.46 \pm 0.50$ & $0.55 \pm 0.30$ & 0.043 \\
\hline NGAL (ng/ml) & $37.8 \pm 53.2$ & $47.8 \pm 70.5$ & 0.551 \\
\hline KIM-1 (pg/ml) & $1.16 \pm 1.20$ & $1.68 \pm 1.67$ & 0.178 \\
\hline
\end{tabular}

